\title{
Executive Functions in Chronic Mesial Temporal Lobe Epilepsy
}

\author{
Laura Zamarian, ${ }^{1}$ Eugen Trinka, ${ }^{1,2}$ Elisabeth Bonatti, ${ }^{1}$ Giorgi Kuchukhidze, ${ }^{1}$ \\ Thomas Bodner, ${ }^{1}$ Thomas Benke, ${ }^{1}$ Florian Koppelstaetter, ${ }^{3}$ and Margarete Delazer ${ }^{1}$ \\ ${ }^{1}$ Clinical Department of Neurology, Innsbruck Medical University, Anichstrasse 35, 6020 Innsbruck, Austria \\ ${ }^{2}$ Department of Neurology, Christian Doppler Clinic, Paracelsus Medical University, Ignaz Herrer Strasse 79, 5020 Salzburg, Austria \\ ${ }^{3}$ Clinical Department of Radiology, Innsbruck Medical University, Anichstrasse 35, 6020 Innsbruck, Austria
}

Correspondence should be addressed to Laura Zamarian, laura.zamarian@uki.at

Received 24 August 2010; Revised 6 December 2010; Accepted 11 January 2011

Academic Editor: Andreas Schulze-Bonhage

Copyright ( 2011 Laura Zamarian et al. This is an open access article distributed under the Creative Commons Attribution License, which permits unrestricted use, distribution, and reproduction in any medium, provided the original work is properly cited.

There is no consensus as to whether mesial temporal lobe epilepsy (MTLE) leads to executive function deficits. In this study, we adopted an extensive neuropsychological test battery and assessed different executive functions in chronic, unilateral MTLE. Performance of MTLE patients was compared with that of healthy peers and with normative data. Several MTLE patients had scores below cut-off or below the 10th percentile of normative data. Scores of the whole patient group were overall in the average range of normative data. Relative to controls, MTLE patients performed poorly in tests of working memory, cognitive flexibility, categorical verbal fluency, set-shifting, categorization, and planning. These findings raise an important methodological issue as they suggest that executive function deficits in chronic MTLE may be individually variable and that their assessment should include different tests. Deficits in chronic MTLE are not limited to temporal lobe functions, such as memory, but may extend to extra temporal cognitive domains, such as executive functions.

\section{Introduction}

Mesial temporal lobe epilepsy (MTLE) is possibly the most frequent form of epilepsy [1] and is associated with overt or subtle structural abnormalities in the hippocampus, parahippocampal gyrus, and amygdala [2]. Recent investigations have suggested that functional and structural abnormalities in MTLE may extend beyond the temporal lobes. Volume reduction has been observed in extratemporal areas such as thalamus, caudate nuclei, lenticular nuclei, corpus callosum, and frontal lobes (for a review, see [3]). Alterations of frontotemporal white matter tracts [4] and of neurotransmitter systems [5] have also been reported. Metabolic changes have been observed not only in mesial and lateral temporal areas, but also in the prefrontal cortex [6] and in subcortical structures $[7,8]$. Thalamus, basal ganglia, and frontal lobes are part of corticosubcortical circuits that are involved in the regulation of motor functions, behavior, and cognitive functions such as set-shifting, planning, and inhibitory control $[9,10]$. As increasing evidence points to functional and structural alterations of these corticosubcortical circuits, it may be expected that MTLE patients experience executive function deficits.

The term "executive functions" refers to high-order cognitive functions which allow adaptation to nonroutine situations such as novel, conflicting, or complex tasks $[11,12]$. Planning, set-shifting, strategic behavior, response initiation, and response inhibition are some of the cognitive functions that may be listed under this umbrella term. Executive function deficits are often found in association with frontal lobe damage $[13,14]$. However, recent neuropsychological investigations as well as neuroimaging studies have pointed to a distributed brain network, which encompasses frontal areas as well as posterior areas (e.g., parietal association areas: [15]; cerebellum: [16]) and subcortical structures (e.g., basal ganglia: [17]; thalamus: [18]). Executive function deficits may be found in a large number of neurological pathologies, in diffuse brain damage, and in focal brain damage [11, 12]. Single-case analyses of brain-damaged patients have shown that executive function deficits may have different 
clinical manifestations and that performance on executive function tests may be dissociated with deficits observed on some tests and not on others $[11,12]$. Executive function deficits may have important consequences on the patient's autonomy and severely compromise the quality of life. Given the multicomponent aspect of executive functioning, its systematic assessment needs to cover several cognitive processes.

Chronic MTLE may affect cognitive and emotional processing [19-21]. Compared with healthy controls, MTLE patients often show reduced episodic memory and difficulties in learning new information [22,23]. Recent studies have also reported difficulties in decision making $[24,25]$. There are mixed results regarding executive functioning in chronic MTLE. Some studies have reported reduced performance for MTLE patients as compared with controls [26-28]; others have found no relevant differences $[22,24]$. As, in most studies, only a single test or a very short test battery has been used to assess executive functioning in MTLE, it may be possible that deficits have been overlooked in some cases. Also, no specific assessment of different executive functions has been done.

Studies that have used the Wisconsin Card Sorting Task (WCST) [42] have found significant differences between MTLE patients and controls, pointing to abstraction, categorization, and set-shifting deficits in MTLE (e.g., [24, 2628]; see also [43]). Studies that have screened for deficits in working memory $[22,24]$, inhibitory control $[24,28]$, verbal fluency $[22,28]$, or cognitive flexibility $[22,24]$ have obtained heterogeneous findings, making it difficult to conclude whether and to what extent MTLE patients present executive function deficits. Inconsistent findings have also been reported with regard to the effect of seizure lateralization. Giovagnoli [26] found that patients with left-sided MTLE are more impaired than patients with right-sided MTLE on the WCST, whereas Corcoran and Upton $[28,44]$ reported the opposite pattern of results. Other investigations found no significant effect of seizure lateralization on executive functioning $[22,45]$.

This study aimed at investigating executive functioning in a group of chronic, unilateral MTLE patients. Differently from previous studies, we used an extensive battery of neuropsychological tests and assessed different aspects of executive functioning (verbal attention, verbal working memory, psychomotor speed, cognitive flexibility, verbal fluency, set-shifting, categorization, inhibition, and planning). In this study, we compared performance of MTLE patients with that of healthy peers as well as with normative data. In separate analyses, we also controlled for possible effects of seizure lateralization (left-sided MTLE versus right-sided MTLE), structural abnormality (hippocampus versus amygdala), and antiepileptic drug therapy (AED), which has been documented to significantly affect cognitive performance [46-49]. A correlation analysis also examined the association of executive function performance with age at seizure onset and epilepsy duration.

Following previous reports on deficits of TLE patients in single executive function tests, we expected the MTLE patients to perform poorly relative to the healthy controls in the more comprehensive neuropsychological test battery. As epilepsy in the dominant hemisphere may have detrimental effects on language processing, we also expected the leftsided MTLE patients to perform poorly relative to the rightsided MTLE patients on verbal fluency tests. Finally, we hypothesized that executive function deficits are enhanced in the patients with the earlier onset epilepsy and the longer disease duration.

\section{Methods}

2.1. Participants. We examined prospectively 28 consecutive patients with unilateral MTLE (12 women). (Patients also performed tasks of decision making; these results are described in [25].) Patients had pharmacoresistant, focal epilepsy and were candidates for epilepsy surgery (age at seizure onset: mean $21.5 \pm 14.5$ years; disease duration: mean $18.2 \pm 15.2$ years). Patients underwent a detailed clinical and neurological examination including prolonged video-EEG monitoring, high-resolution MRI, interictal SPECT or PET, and a neuropsychological examination. They were seizurefree for at least 48 hours before the neuropsychological testing. Twenty-six patients were right hander [50]; two were ambidextrous (only right-hander patients were taken into consideration in the analysis of the effect of seizure lateralization).

Patients underwent a high-resolution 1.5-Tesla MRI with T1-weighted spin echo and gradient echo 3D multiplanar reconstruction images (with and without intravenous contrast application), axial and coronal T2-weighted turbospin echo, T1 inversion recovery, T2-weighted fast fluid attenuated inversion recovery (FLAIR), and diffusion-weighted sequences. Coronal T2-weighted and FLAIR slices extended $1-3 \mathrm{~mm}$. Axial images were acquired with an angle parallel to the long axis of the hippocampus. All but three MRI scans were visually evaluated by two independent raters (G. K., F. K.) who were familiar with the radiological analysis of the temporal lobe structures and were blinded to diagnosis. Three patients underwent MRI outside our institution following the above-mentioned protocol. For these three patients, we obtained a detailed written description of the mesial temporal structures. Agreement was achieved in all but two cases (agreement: 93\%). A third rater (E.T.) who was blinded to diagnosis was consulted to resolve these two cases. MRI revealed a clear unilateral abnormality in the left mesial temporal lobe in 17 patients (LMTLE) and a clear unilateral abnormality in the right mesial temporal lobe in 11 patients (RMTLE). Hippocampal abnormality was found in 10 patients (hippocampus group = MTLE-HC), amygdala abnormality in 11 patients (corpus amygdaloideum group = MTLE-CA), and abnormalities affecting both structures in 7 patients (see Figure 1 for examples of MRI features for the three groups). The MTLE-HC group included patients with MRI features of hippocampal sclerosis (atrophic hippocampus with missing internal three-layer architecture and increased signal in T2 and FLAIR sequences) and hippocampal dysplasia (hippocampus with distorted anatomy, with blurring of white/grey matter interface, and increased signal in T2 and FLAIR sequences). Amygdala was regarded as dysplastic if it 


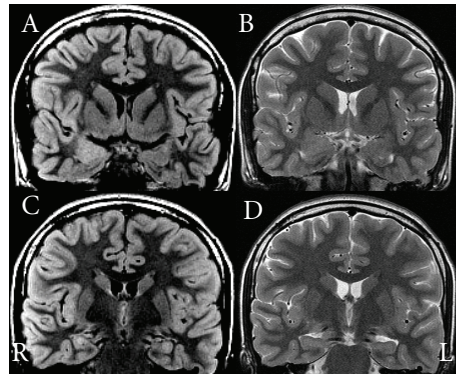

(a) Patient AJ (MTLE-HC group)

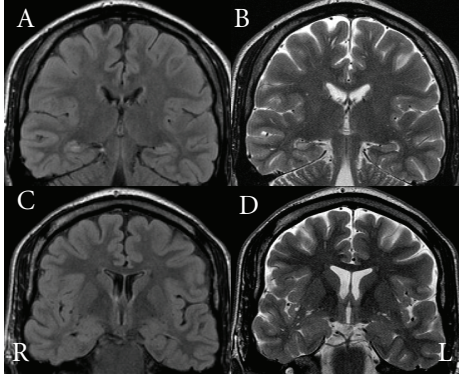

(b) Patient PE (MTLE-CA group)

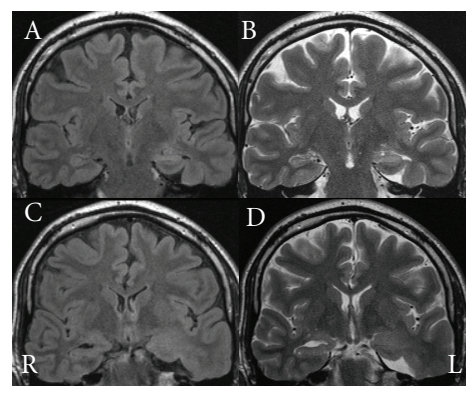

(c) Patient WE (MTLE-HC+CA group)

Figure 1: Examples of MRI features for the MTLE-HC group (a), the MTLE-CA group (b), and the MTLE-HC+CA group (c). (a) Upper row: (A) FLAIR and (B) T2-weighted coronal images showing on the right side enlarged amygdala with increased MR signal in FLAIR and T2-weighted sequences (dysplastic amygdala). Lower row: (C) FLAIR and (D) T2-weighted coronal images showing normal hippocampus; R: right hemisphere; L: left hemisphere. (b) Upper row: (A) FLAIR and (B) T2-weighted coronal images showing on the right side atrophic hippocampus with increased MR signal in FLAIR and T2-weighted sequences (hippocampal sclerosis). Lower row: (C) FLAIR and (D) T2-weighted coronal images showing normal amygdala; R: right hemisphere; L: left hemisphere. (c) Upper row: (A) FLAIR and (B) T2weighted coronal images showing on the left side dysplastic hippocampus with increased MR signal in FLAIR and T2-weighted sequences. Lower row: (C) FLAIR and (D) T2-weighted coronal images showing on the left side enlarged amygdala with increased MR signal in FLAIR and T2-weighted sequences (dysplastic amygdala); R: right hemisphere; L: left hemisphere.

was larger than the contralateral side, if increased signal in T2 and FLAIR sequences was observed, or both.

Fifteen patients received antiepileptic drug monotherapy (carbamazepine, oxcarbazepine, lamotrigine, and levetiracetam); 13 patients were on polytherapy (carbamazepine, lamotrigine, levetiracetam, oxcarbazepine, phenobarbital, phenytoin, pregabalin, primidone, topiramate, valproate, zonisamide; AED daily doses and serum levels are reported for each patient in Table 4). The serum level was measured within one day from the neuropsychological examination in $24 / 28$ patients ( $86 \%$ ); in the remaining cases, the serum level refers to the closest previous measurement (deviation from the day of the neuropsychological examination: 17 to 44 days). All but one patient had serum levels within standards. The patient with the serum level for one antiepileptic drug slightly above the normal range did not manifest signs of intoxication. Also, neuropsychological performance did not differ between this patient and the patient group.

Twenty healthy volunteers ( 15 women) participated in the study. They were recruited from the personnel of the Innsbruck Medical University. Exclusion criteria for participation were history of stroke, head trauma, psychiatric disorder, substance abuse, or neurological disorders which may compromise cognition. Healthy participants and MTLE patients were comparable in age (controls: mean $36.0 \pm 13.0$ years; MTLE patients: mean $40.3 \pm 11.3$ years) and education (controls: mean $11.4 \pm 1.6$ years; MTLE patients: mean 10.6 \pm 1.7 years). The present study was approved by the local ethics committee. Informed consent was obtained from all participants.

2.2. Neuropsychological Assessment. MTLE patients completed tests of verbal memory (learning, free recall, and recognition-Münchner Gedächtnistest, MGT) [33], nonverbal memory (subtest of the Visueller und Verbaler Merkfähigkeitstest, VVM) [34], vocabulary [29], numeracy $[30,31]$, mental complex calculation [32], and executive functions (verbal attention and verbal working memorydigit span tests of the Wechsler Memory Scale-Revised, WMS-R [36]; psychomotor speed and cognitive flexibilityTrail Making Test-A and B, TMT-A and B [37]; categorical verbal fluency, phonological verbal fluency, and verbal fluency with alternating categories-subtests of the Regensburger Wortflüssigkeitstest, RWT [38]; planning and problem solving-Planungstest [41]; abstraction and categorization-WCST [39]; inhibitory control-Go-NoGo task adapted from [40]). MTLE patients also responded to a questionnaire on anxiety and depression (Hospital Anxiety and Depression Scale-Deutsche Version, HADS-D) [35]. Controls were tested on vocabulary, numeracy, mental complex calculation, and executive functions. The numeracy test assesses basic probability and mathematical concept understanding $[30,31]$.

2.3. Statistical Analysis. Data did not satisfy assumptions of a normal distribution. Therefore, group differences were analyzed through nonparametric methods (Kruskal-Wallis $\mathrm{H}$ test, Mann-Whitney $U$-test). Significance was set at $\alpha=.05$.

A Spearman-rank correlation analysis was carried out for the MTLE group between executive function scores, age at seizure onset, and epilepsy duration. The following executive function variables were entered into the analysis: completed categories (WCST), perseverative errors (WCST), digit span backward (WMS-R), correct NoGo trials (Go-NoGo task), sec in the TMT-B, total number of moves (Planungstest), categorical verbal fluency (RWT), and verbal fluency with alternating categories (set-shifting, RWT). Applying Bonferroni correction for multiple correlations, significance was set at $\alpha=.003$.

\section{Results}

3.1. Comparison with Normative Data. Median scores of healthy controls were in the average range of normative data 
TABLE 1: Medians for scores in neuropsychological background tests as a function of group.

\begin{tabular}{|c|c|c|c|c|c|c|}
\hline & \multirow{2}{*}{$\begin{array}{l}\text { Max. } \\
\text { score }\end{array}$} & \multirow{2}{*}{$\begin{array}{c}\text { MTLE } \\
\text { patients } \\
\text { Mdn }\end{array}$} & \multirow{2}{*}{$\begin{array}{c}\text { Healthy } \\
\text { controls } \\
\text { Mdn }\end{array}$} & \multirow{2}{*}{$P$ value } & \multicolumn{2}{|c|}{ Patients deviating from norms } \\
\hline & & & & & $(n / N)$ & $(\%)$ \\
\hline Vocabulary [29] & 37 & 28.5 & 31.0 & .010 & $0 / 28^{1}$ & 0 \\
\hline Numeracy $[30,31]$ & 11 & $8.0^{*}$ & 9.0 & .004 & $15 / 28^{2}$ & 54 \\
\hline Mental complex calculation [32] & 24 & 11.0 & 14.0 & .044 & $0 / 23^{3}$ & 0 \\
\hline \multicolumn{7}{|l|}{ Verbal memory (MGT) [33] } \\
\hline Learning (5. trial) & 16 & 13.0 & - & - & $5 / 27^{4}$ & 19 \\
\hline Immediate free recall & 16 & 10.0 & - & - & $11 / 27^{4}$ & 41 \\
\hline Delayed free recall & 16 & 12.0 & - & - & $9 / 27^{4}$ & 33 \\
\hline Recognition (corrected score) & 16 & 15.0 & - & - & $8 / 27^{4}$ & 30 \\
\hline $\begin{array}{l}\text { Nonverbal memory, immediate free recall } \\
(\mathrm{VVM})[34]\end{array}$ & 31 & 18.0 & - & - & $6 / 25^{4}$ & 24 \\
\hline Anxiety (HADS-D) [35] & 21 & 6.0 & - & - & $5 / 28^{5}$ & 18 \\
\hline Depression (HADS-D) [35] & 21 & 2.0 & - & - & $2 / 28^{5}$ & 7 \\
\hline Digit span forward (WMS-R) [36] & 12 & 7.0 & 7.5 & .069 & $7 / 28^{4}$ & 25 \\
\hline Digit span backward (WMS-R) [36] & 12 & 6.0 & 7.0 & .013 & $8 / 28^{4}$ & 29 \\
\hline Psychomotor speed (TMT-A) [37] & & 28.5 & 28.0 & .379 & $3 / 28^{4}$ & 11 \\
\hline Cognitive flexibility (TMT-B) [37] & & 77.0 & 55.5 & .007 & $12 / 28^{4}$ & 43 \\
\hline Categorical verbal fluency (RWT) [38] & & 23.5 & 27.0 & .027 & $8 / 28^{4}$ & 29 \\
\hline Phonological verbal fluency (RWT) [38] & & 7.0 & 9.0 & .080 & $12 / 27^{4}$ & 44 \\
\hline Set-shifting (RWT) $[38]$ & & 14.0 & 16.0 & .004 & $2 / 28^{4}$ & 7 \\
\hline \multicolumn{7}{|l|}{ Categorization (WCST) [39] } \\
\hline Completed categories & 5 & 2.5 & 4.0 & .010 & $7 / 28^{4}$ & 25 \\
\hline Perseverative errors & & 10.0 & 6.0 & .011 & $10 / 28^{4}$ & 36 \\
\hline \multicolumn{7}{|l|}{ Go-NoGo task (adapted from [40]) } \\
\hline Correct Go-trials & 100 & 98.9 & 100.0 & .038 & $2 / 28^{4}$ & 7 \\
\hline Correct NoGo-trials & 100 & 83.3 & 80.0 & .892 & $3 / 28^{4}$ & 11 \\
\hline \multicolumn{7}{|l|}{ Planning (Planungstest) [41] } \\
\hline Total number of moves & & 36.0 & 34.0 & .009 & $2 / 28^{4}$ & 7 \\
\hline Errors & & 0.0 & 0.0 & - & $1 / 28^{4}$ & 4 \\
\hline
\end{tabular}

Note: Group comparisons were carried out by means of Mann-Whitney $U$-test. Significance was set at $\alpha=.05$.

MTLE: mesial temporal lobe epilepsy; Mdn: Median; $\left({ }^{1}\right)$ : cut-off IQ 85; $\left({ }^{2}\right)$ : cut-off median 9; $\left({ }^{3}\right)$ : cut-off 5; $\left({ }^{4}\right)$ : cut-off 10 th percentile; $\left({ }^{5}\right)$ : cut-off $10 ;(*)$ : slightly below cut-off; (-): not available.

in all tests (Tables 1 and 2). The MTLE patients' median score in the numeracy test was below cut-off. In other tests, the MTLE patients' median scores were in the average range of normative data. The evaluation of individual scores revealed several patients with performance below cut-off or below the 10th percentile of normative data (4-54\%; Tables 1 and 2). Regarding the executive function tests, 7 patients $(25 \%)$ performed below the 10th percentile of normative data in 5to- 8 measures; 19 patients $(68 \%)$ performed below the 10 th percentile in 1-to-3 measures. Only two patients showed a normal performance in all measures.

3.2. Comparison of MTLE Patients with Healthy Controls. Performance of MTLE patients was compared with that of healthy controls by means of Mann-Whitney $U$-test. Group differences were significant in several neuropsychological measures. MTLE patients obtained slightly lower vocabulary scores, numeracy scores, and mental complex calculation scores than controls (Table 1). Relative to controls, MTLE patients also performed poorly in tests of verbal working memory, cognitive flexibility, categorical verbal fluency, setshifting, categorization, and planning (Table 1). A significant group difference was also found in the Go-NoGo task. MTLE patients responded less accurately than controls to Go trials; groups did not differ from each other in NoGo trials. There were no significant group differences in tests of verbal attention, psychomotor speed, and phonological verbal fluency (Table 1).

\subsection{Effect of Seizure Lateralization}

3.3.1. Comparison with Normative Data. RMTLE patients had a median score slightly below cut off in the numeracy test and a median score slightly below the 10th percentile 
TABLE 2: Medians for demographic information and scores in neuropsychological background tests as a function of seizure lateralization.

\begin{tabular}{|c|c|c|c|c|}
\hline & $\begin{array}{l}\text { Max. } \\
\text { score }\end{array}$ & $\begin{array}{l}\text { LMTLE patients } \\
\qquad(n=16)\end{array}$ & $\begin{array}{l}\text { RMTLE patients } \\
\quad(n=10)\end{array}$ & $P$ value \\
\hline & & Mdn & Mdn & \\
\hline Age (years) & & 46.0 & 35.5 & .076 \\
\hline Education (years) & & 10.0 & 10.0 & .075 \\
\hline Age at disease onset (years) & & 20.5 & 17.0 & - \\
\hline Disease duration (years) & & 11.5 & 14.0 & - \\
\hline Vocabulary [29] & 37 & 27.0 & 29.0 & .057 \\
\hline Numeracy $[30,31]$ & 11 & $8.5^{*}$ & $7.5^{*}$ & .014 \\
\hline Mental complex calculation [32] & 24 & 11.5 & 9.0 & .034 \\
\hline \multicolumn{5}{|l|}{ Verbal memory (MGT) [33] } \\
\hline Learning (5. trial) & 16 & 13.0 & 15.0 & - \\
\hline Immediate free recall & 16 & $9.5^{*}$ & 13.0 & - \\
\hline Delayed free recall & 16 & 12.0 & 14.0 & - \\
\hline Recognition (corrected score) & 16 & 15.0 & 14.0 & - \\
\hline $\begin{array}{l}\text { Nonverbal memory, immediate free recall } \\
(\text { VVM) [34] }\end{array}$ & 31 & 17.5 & 17.0 & - \\
\hline Anxiety (HADS-D) [35] & 21 & 5.5 & 7.0 & - \\
\hline Depression (HADS-D) [35] & 21 & 4.5 & 2.0 & - \\
\hline Digit span forward (WMS-R) [36] & 12 & 7.0 & 6.0 & .079 \\
\hline Digit span backward (WMS-R) [36] & 12 & 5.5 & 5.5 & .047 \\
\hline Psychomotor speed (TMT-A) [37] & & 29.5 & 24.5 & .691 \\
\hline Cognitive flexibility (TMT-B) [37] & & 78.0 & 73.5 & .018 \\
\hline Categorical verbal fluency (RWT) [38] & & 22.5 & 27.0 & .056 \\
\hline Phonological verbal fluency (RWT) [38] & & $5.0^{*}$ & 8.0 & .012 \\
\hline Set-shifting (RWT) [38] & & 13.5 & 15.0 & .011 \\
\hline \multicolumn{5}{|l|}{ Categorization (WCST) [39] } \\
\hline Completed categories & 5 & 3.5 & $2.0^{*}$ & .027 \\
\hline Perseverative errors & & 10.0 & 10.0 & .032 \\
\hline \multicolumn{5}{|l|}{ Go-NoGo task (adapted from [40]) } \\
\hline Correct Go-trials & 100 & 98.9 & 98.9 & .059 \\
\hline Correct NoGo-trials & 100 & 83.3 & 98.6 & .878 \\
\hline \multicolumn{5}{|l|}{ Planning (Planungstest) [41] } \\
\hline Total number of moves & & 35.5 & 36.0 & .029 \\
\hline Errors & & 0.0 & 0.0 & - \\
\hline
\end{tabular}

Note: Group comparisons between LMTLE patients, RMTLE patients, and healthy controls were carried out by means of Kruskal-Wallis $H$-test. Significance was set at $\alpha=.05$.

LMTLE: left-sided mesial temporal lobe epilepsy; RMTLE: right-sided mesial temporal lobe epilepsy; Mdn: Median; $(*)$ : slightly below cut-off or below the 10th percentile of normative data; (-): not available.

in the WCST (Table 2). LMTLE patients had a median score slightly below cut off in the numeracy test and a median score slightly below the 10th percentile in the verbal memory test (immediate free recall) and the phonological verbal fluency test (Table 2). In other tests, the median scores of both groups were in the average range of normative data.

3.3.2. Comparison with Healthy Controls. We performed group comparisons by means of Kruskal-Wallis $H$-tests. As indicated in Table 2, results were significant in tests of numeracy, mental complex calculation, verbal working memory, cognitive flexibility, phonological verbal fluency, set-shifting, categorization, and planning. Two-by-two comparisons were carried out by means of Mann-Whitney $U$ tests. LMTLE patients obtained lower scores than RMTLE patients in the phonological verbal fluency test $(z=-2.06$, $P=.041$ ); other differences between the patient groups were not significant. Relative to healthy controls, LMTLE patients performed poorly in tests of cognitive flexibility $(z=-2.68$, $P=.007)$, phonological verbal fluency $(z=-2.81, P=$ $.004)$, set-shifting $(z=-3.01, P=.002)$, categorization (completed categories: $z=-2.35, P=.019$; perseverative errors: $z=-2.46, P=.014)$, and planning $(z=-2.16, P=$ .033). RMTLE patients obtained lower scores than healthy 
TABLE 3: Medians for demographic information and scores in neuropsychological background tests as a function of structural abnormality.

\begin{tabular}{|c|c|c|c|c|}
\hline & $\begin{array}{l}\text { Max. } \\
\text { score }\end{array}$ & $\begin{array}{l}\text { MTLE-HC patients } \\
\quad(n=10)\end{array}$ & $\begin{array}{l}\text { MTLE-CA patients } \\
\quad(n=11)\end{array}$ & $P$ value \\
\hline & & Mdn & Mdn & \\
\hline Age (years) & & 47.0 & 34.0 & .017 \\
\hline Education (years) & & 9.5 & 10.0 & .001 \\
\hline Age at disease onset (years) & & 17.5 & 15.0 & - \\
\hline Disease duration (years) & & 30.5 & 6.0 & - \\
\hline Vocabulary [29] & 37 & 25.0 & 29.0 & .030 \\
\hline Numeracy $[30,31]$ & 11 & $7.0^{*}$ & $8.0^{*}$ & .059 \\
\hline Mental complex calculation [32] & 24 & 9.0 & 12.5 & .310 \\
\hline \multicolumn{5}{|l|}{ Verbal memory (MGT) [33] } \\
\hline Learning (5. trial) & 16 & 12.0 & 14.0 & - \\
\hline Immediate free recall & 16 & $6.5^{*}$ & 13.0 & - \\
\hline Delayed free recall & 16 & $8.5^{*}$ & 14.0 & - \\
\hline Recognition (corrected score) & 16 & $12.0^{*}$ & 16.0 & - \\
\hline $\begin{array}{l}\text { Nonverbal memory, immediate free recall } \\
(\mathrm{VVM})[34]\end{array}$ & 31 & 16.0 & 21.0 & - \\
\hline Anxiety (HADS-D) [35] & 21 & 9.5 & 6.0 & - \\
\hline Depression (HADS-D) [35] & 21 & 3.5 & 2.0 & - \\
\hline Digit span forward (WMS-R) [36] & 12 & 6.0 & 7.0 & .128 \\
\hline Digit span backward (WMS-R) [36] & 12 & 5.0 & 6.0 & .031 \\
\hline Psychomotor speed (TMT-A) [37] & & 33.0 & 27.0 & .866 \\
\hline Cognitive flexibility (TMT-B) [37] & & 80.5 & 71.0 & .078 \\
\hline Categorical verbal fluency (RWT) [38] & & 20.0 & 27.0 & .326 \\
\hline Phonological verbal fluency (RWT) [38] & & 8.0 & 8.0 & .746 \\
\hline Set-shifting (RWT) [38] & & 13.0 & 14.0 & .187 \\
\hline \multicolumn{5}{|l|}{ Categorization (WCST) [39] } \\
\hline Completed categories & 5 & $1.0^{*}$ & 4.0 & .014 \\
\hline Perseverative errors & & $17.5^{*}$ & 9.0 & .047 \\
\hline \multicolumn{5}{|l|}{ Go-NoGo task (adapted from [40]) } \\
\hline Correct Go-trials & 100 & 98.3 & 98.9 & .646 \\
\hline Correct NoGo-trials & 100 & $73.3^{*}$ & 86.7 & .205 \\
\hline \multicolumn{5}{|l|}{ Planning (Planungstest) [41] } \\
\hline Total number of moves & & 36.0 & 36.0 & .416 \\
\hline Errors & & 0.0 & 0.0 & - \\
\hline
\end{tabular}

Note: Group comparisons between MTLE-HC patients, MTLE-CA patients, and healthy controls were carried out on residualized dependent variables by means of Kruskal-Wallis $H$-test. Significance was set at $\alpha=.05$.

MTLE-HC: patients with hippocampal abnormalities; MTLE-CA: patients with abnormalities in the corpus amygdaloideum; Mdn: Median; $(*)$ : slightly below cut-off or below the 10th percentile of normative data; (-): not available.

controls in tests of numeracy $(z=-2.86, P=.005)$, mental complex calculation $(z=-2.30, P=.019)$, verbal working memory $(z=-2.33, P=.022)$, categorization (completed categories: $z=-2.15, P=.035)$, and planning $(z=-2.24$, $P=.028)$. Other differences between patient groups and healthy controls were not significant.

3.3.3. Further Comparisons between RMTLE Patients and LMTLE Patients. Mann-Whitney $U$-tests indicated that RMTLE patients and LMTLE patients were comparable in terms of age at seizure onset and epilepsy duration. There were also no significant group differences in tests of verbal memory, nonverbal memory, anxiety, and depression.

\subsection{Effect of Structural Abnormality}

3.4.1. Comparison with Normative Data. MTLE-CA patients scored slightly below cut-off in the numeracy test (Table 3 ). MTLE-HC patients scored below cut-off in the numeracy test and below the 10th percentile in tests of verbal memory, categorization, and inhibitory control (Table 3 ). Other median scores were in the average range of normative data for both patient groups. 
3.4.2. Comparison with Healthy Controls. As the MTLE-HC patients were older and had less education than both the healthy controls and the MTLE-CA patients, we residualized age and education on the dependent variables and then used these measures in the analysis. Group comparisons were performed by means of Kruskal-Wallis $H$-tests. There were significant group differences in tests of vocabulary, verbal working memory, and categorization (Table 3). MTLE-HC patients obtained lower scores than MTLE-CA patients in the WCST (completed categories: $z=-1.97, P=.051$ ). Relative to healthy controls, MTLE-HC patients performed poorly in tests of vocabulary $(z=-1.98, P=.005)$, verbal working memory $(z=-2.68, P=.006)$, and categorization (completed categories: $z=-2.75, P=.005$; perseverative errors: $z=-2.48, P=.012$ ). Differences between MTLE-CA patients and healthy controls were not significant.

3.4.3. Further Comparisons between MTLE-HC Patients and MTLE-CA Patients. As indicated by Mann-Whitney $U$-test, MTLE-HC patients and MTLE-CA patients had comparable age at seizure onset. The MTLE-HC group had longer epilepsy duration than the MTLE-CA group $(z=-2.44, P=$ .012). Differences between MTLE-HC patients and MTLECA patients in tests of verbal memory, nonverbal memory, anxiety, and depression were not significant when residualized scores were submitted to Mann-Whitney $U$-tests.

3.5. Effect of Antiepileptic Drug Therapy. Patients on monotherapy and patients on polytherapy had comparable scores in all demographic, clinical, and neuropsychological measures (Mann-Whitney $U$-tests, all $P>.05$ ). The comparison of individual scores with the patient group distribution indicated that five patients had an outlier performancethat is, below $2.5 \mathrm{SD}$ from the group mean-in some memory and executive function measures. Outlier cases were evenly distributed between the monotherapy group and the polytherapy group (see Table 4).

3.6. Correlation Analysis. There was a significant correlation between epilepsy duration and performance on the WCST (completed categories, $r=-.581, P=.002$ ). The longer the disease duration, the poorer the performance. No significant correlation was found between age at seizure onset and performance on executive function tests. (An exploratory analysis indicated that performance on nonexecutive function tests (vocabulary, memory, and complex numerical processing) directly correlated with performance on executive function tests. However, these results did not survive Bonferroni correction for multiple correlations. Neither performance on executive function tests nor performance on nonexecutive function tests correlated with anxiety and depression scores.)

\section{Discussion}

This study used an extensive neuropsychological test battery and assessed several aspects of executive functioning in chronic, unilateral MTLE patients. Performance of a carefully selected group of pharmacoresistant MTLE patients, who were candidates for epilepsy surgery, was compared with that of healthy peers as well as with normative data. Possible effects of seizure lateralization, structural abnormality, antiepileptic drug therapy, age at seizure onset, and epilepsy duration were also analyzed.

Results indicated that both the MTLE group and the healthy control group had median scores in the average range of normative data. However, there were significant group differences in several executive function measures. Relative to healthy peers, MTLE patients performed poorly in tests of verbal working memory, cognitive flexibility, categorical verbal fluency, set-shifting, categorization, planning, and inhibitory control. They performed comparably to healthy controls in tests of verbal attention, psychomotor speed, and phonological verbal fluency. The evaluation of individual scores corroborated the results of these group comparisons. Several patients had scores below cut-off or below the 10th percentile of normative data. Also, almost one-third of the patients had impairments in 5-to- 8 measures of executive functioning; two thirds had impairments in 1-to-3 measures. These findings raise an important methodological issue as they suggest that executive function deficits in chronic MTLE may be individually variable and that their assessment needs to include different tests. We suggest the use of an extensive neuropsychological test battery which assesses different aspects of executive functioning (e.g., working memory, cognitive flexibility, categorization, inhibitory control, and planning). As shown by this study, the use of a single task (e.g., Go-NoGo) is not sufficient for the detection of executive function deficits which may be highly specific.

As a correlation analysis indicated, performance of MTLE patients on the WCST inversely correlated with epilepsy duration. Similar results were described by Kim et al. [27], whereas Martin et al. [43] found no association between performance on the WCST and epilepsy duration or age at seizure onset. Black et al. [45] tested working memory and executive functions in a large group of TLE patients (the majority of patients had mesial temporal lobe epilepsy). Results showed that age at seizure onset and seizure frequency, which significantly correlated with epilepsy duration, were significant predictors of the patients' impairments. In this study, MTLE patients had intractable epilepsy, and epilepsy duration ranged from few months to several years. As the brain suffers for longer time from the epileptic discharges [51], it seems likely that the cognitive deficits were more pronounced in the MTLE patients with the longer disease duration.

The negative effect of epilepsy duration on cognition may be related not only to the effect of the epileptic seizures themselves, but also to other factors including the long-term antiepileptic drug therapy. All commonly used antiepileptic drugs seem to have some behavioral and cognitive effects, with the patients on polytherapy being on average more affected than the patients on monotherapy [46-49]. For example, phenytoin, phenobarbital, and valproate have been related to decline in attention and psychomotor speed (for a review, see [48]); also, patients taking topiramate have been found to perform poorly on verbal fluency [52] 
TABLE 4: Individual daily dose and serum level of the antiepileptic drug therapy.

\begin{tabular}{|c|c|c|c|c|c|c|}
\hline Patient code & Gender & Age & Education & AED daily dose (mg) & AED serum level $(\mu \mathrm{g} / \mathrm{ml})$ & Outlier \\
\hline P 1 & $\mathrm{~m}$ & 35 & 10 & PGB 600, PB 150 & PGB 6.3, PB 11.1 & \\
\hline P 2 & $\mathrm{~m}$ & 34 & 10 & LEV 2000, PHE 200 & LEV 9.2, PHE 3.2 & \\
\hline P 3 & $\mathrm{f}$ & 46 & 10 & LTG 150, LEV 2000 & LTG 2.0, LEV 27.4 & $\mathrm{a}$ \\
\hline $\mathrm{P} 4$ & $\mathrm{f}$ & 48 & 9 & LTG 400, PRM 500, ZNS 200 & LTG 9.5, PB 12.0, PRM 10.4, ZNS 18.6 & $b, c, d$ \\
\hline P 5 & $\mathrm{~m}$ & 49 & 9 & CBZ RD 1050 & CBZ 3.8, CBZ Epoxid 0.6 & \\
\hline P 6 & $\mathrm{f}$ & 44 & 12 & CBZ RD 600 & CBZ 7.7 & \\
\hline P 7 & $\mathrm{~m}$ & 46 & 10 & CBZ 300 & CBZ 3.4 & \\
\hline P 8 & $\mathrm{~m}$ & 20 & 13 & OXC 900 & OXC 0.4, 10-OH-Carbazepin 24.6 & \\
\hline P 9 & $\mathrm{~m}$ & 44 & 10 & OXC 900, LEV 3000 & OXC 0.2, 10-OH-Carbazepin 13.2, LEV 27.0 & \\
\hline P 10 & $\mathrm{f}$ & 46 & 10 & LEV 3000 & LEV 47.9 & $\mathrm{e}$ \\
\hline P 11 & $\mathrm{~m}$ & 37 & 13 & CBZ 300 & CBZ 0.0 & \\
\hline P 12 & $\mathrm{~m}$ & 33 & 13 & CBZ RD 1200 & CBZ 7.9 & \\
\hline P 13 & $\mathrm{~m}$ & 59 & 10 & CBZ RD 200 & CBZ 1.7, CBZ Epoxid 0.1 & $\mathrm{~d}, \mathrm{f}$ \\
\hline P 14 & $\mathrm{~m}$ & 51 & 9 & CBZ RD 1200, TPM 250 & CBZ 10.1, TPM 4.5 & \\
\hline P 15 & $\mathrm{f}$ & 24 & 10 & CBZ 750 & CBZ 8.6 & a \\
\hline P 16 & $\mathrm{f}$ & 54 & 9 & LEV 1500 & LEV 28.8 & \\
\hline P 17 & $\mathrm{~m}$ & 58 & 10 & CBZ RD 800, ZNS 200 & CBZ 10.1, CBZ Epoxid 1.2, ZNS 5.4 & \\
\hline P 18 & $\mathrm{f}$ & 50 & 9 & LEV 2000 & LEV 32 & \\
\hline P 19 & $\mathrm{f}$ & 18 & 12 & LEV 3000, VPA RD 1250, ZNS 300 & LEV 70.3, VPA 111.7, ZNS 22.4 & \\
\hline P 20 & $\mathrm{~m}$ & 38 & 10 & LTG 300, PHE 200 & LTG 3.9, PHE 5.4 & \\
\hline P 21 & $\mathrm{f}$ & 36 & 10 & LTG 200 & LTG 10.7 & \\
\hline P 22 & $\mathrm{~m}$ & 24 & 16 & LEV 3000, PGB 300 & LEV 43.8, PGB 3.9 & \\
\hline P 23 & $\mathrm{~m}$ & 29 & 10 & LEV 3000, PHE 400 & LEV 27.5, PHE 19.9 & \\
\hline P 24 & $\mathrm{f}$ & 35 & 13 & CBZ RD 600 & CBZ 5.8 & \\
\hline P 25 & $\mathrm{~m}$ & 57 & 10 & CBZ RD 400, LEV 3000 & CBZ 5.4, CBZ Epoxid 0.9, LEV 38.2 & \\
\hline P 26 & $\mathrm{~m}$ & 43 & 10 & LEV 4000, OXC 2100 & LEV 23.8, OXC 0.0, 10-OH-Carbazepin 24.3 & \\
\hline P 27 & $\mathrm{f}$ & 30 & 10 & LEV 1000 & LEV 3.4 & \\
\hline P 28 & $\mathrm{f}$ & 40 & 9 & CBZ RD 1400 & CBZ 12.2 & \\
\hline
\end{tabular}

AED: antiepileptic drug; m: male; f: female; CBZ: carbamazepine; LTG: lamotrigine; LEV: levetiracetam; OXC: oxcarbazepine; PB: phenobarbital; PHE: phenytoin; PGB: pregabalin; PRM: primidone; TPM: topiramate; VPA: valproate; ZNS: zonisamide; a: outlier performance in planning (total number of moves or errors, Planungstest); b: outlier performance in categorical verbal fluency (animals/min, RWT); c: outlier performance in categorization (perseverative errors, WCST); d: outlier performance in verbal memory (recognition, MGT); e: outlier performance in verbal attention (digit span forward, WMS-R); f: outlier performance in inhibitory control (correct NoGo trials, Go-NoGo task).

and categorization [27]. Compared with older antiepileptic drugs, some of the new antiepileptic drugs have fewer negative effects on cognition $[46,48]$. In this study, we found that only few patients had an outlier performance relative to performance of the whole patient group. None of the medications seemed to have stronger effects on cognition than others. Also, there were no differences between patients on monotherapy and patients on polytherapy in any memory or executive function measure. Although we cannot exclude the possibility that small group differences between patients on monotherapy and patients on polytherapy have been missed because of the small sample group, these findings may suggest that the antiepileptic drug therapy had no relevant effect on the executive function performance of MTLE patients in this study.

Results of the comparison of left-sided MTLE patients with right-sided MTLE patients showed no relevant group differences with the only exception of a verbal fluency measure. Relative to right-sided MTLE patients, leftsided MTLE patients performed poorly in a phonological verbal fluency test. These results are in line with other investigations, which suggest no severe effect of seizure lateralization on executive functioning [22, 43, 45]. For example, Tudesco et al. [22] tested MTLE patients with unilateral hippocampal sclerosis. Comparably to this study, they found that left-sided MTLE patients differed from right-sided MTLE patients in verbal fluency tests, but not in working memory and set-shifting tests.

There are competing hypotheses in the literature as to the underlying mechanisms of executive function deficits in MTLE. A first hypothesis (nociferous cortex hypothesis) [53] proposes that executive function deficits in MTLE result from the propagation of the epileptic discharges from the temporal lobe epileptic focus to the frontal lobes. There are white matter tracts connecting the temporal lobes with the frontal lobes $[54,55]$, and the epileptic discharges may propagate 
through these projections. According to a second hypothesis (hippocampal contribution hypothesis) [28], executive function deficits in MTLE are related to dysfunction of the hippocampus. Specifically, as the hippocampus is involved in the retrieval of information from short-term memory, in the formation of memory associations, and in learning new information, the MTLE patients' impairments in working memory tasks as well as in other executive function tasks, such as the WCST, that rely on such processes would result from dysfunction of the hippocampus. Finally, increasing evidence [3] points to the implication of extratemporal functional and structural brain abnormalities-for example, damage to frontosubcortical circuits, volume loss, or metabolic changes in extratemporal regions-in the executive function disorders of TLE patients. Our study design does not allow us to precisely define the impact of these different factors on executive function performance. Although an exploratory analysis indicated that patients with hippocampal abnormalities (MTLE-HC) scored lower than patients with amygdala abnormalities (MTLE-CA) and healthy controls in the WCST, we may not exclude the possibility that different factors-propagation of epileptic discharges, dysfunction of mesial temporal structures, and extratemporal brain modifications-contributed to the executive function disorders of MTLE patients in this study. Other investigation methods-for example, functional magnetic resonance imaging, voxel-based morphometry, or diffusion tensor imaging - have to be employed, together with neuropsychological testing, to address this question.

One could ask whether there is a specific pattern in the executive function deficits of MTLE patients. Miyake et al. [56] used a latent variable analysis to investigate how executive functions relate to each other. Results indicated that functions such as updating, shifting, and inhibition are clearly separable, but that they are not completely independent from each other and seem to share some common processes. Miyake et al. [56] proposed that the central executive component of working memory [57] is a feasible candidate to account for some of the commonality underlying different executive functions $[56,58]$. The central executive is an attentional control system, which allocates resources and coordinates and monitors short-term storage processes as well as more general processes [59]. Several executive function tests clearly load on central executive resources, as they require the maintenance of goal and content information, the manipulation of memory representations, or the suppression of irrelevant information. In this study, MTLE patients had difficulties in particular in the tests that put high demands on attentional control resources (e.g., WCST, planning test, and verbal fluency test with alternating categories), whereas they performed relatively well in the less demanding tests (e.g., digit span forward, TMT-A).

In sum, this study indicates that deficits in chronic MTLE are not limited to temporal lobe functions, such as memory, but may extend to extratemporal cognitive domains. Patients with chronic, pharmacoresistant MTLE have executive function deficits, and these deficits are individually variable. Executive functions such as cognitive flexibility, planning, and inhibitory control are essential in everyday life. It is thus possible that MTLE patients experience difficulties in everyday functioning, in particular in those situations that are unknown and require rapid adaptation or flexible problem solving. This study adds to the characterization of cognitive deficits in MTLE and suggests the use of a comprehensive assessment battery in the individual neuropsychological diagnosis.

\section{Acknowledgments}

L. Zamarian received research support from MFI (Medizinische Forschungsförderung, Innsbruck), Medical University Innsbruck, Project No. 2007-419; E. Bonatti received research support from TWF (Tiroler Wissenschaftsfonds), Project No. UNI-0407/29. This study was supported by FWF (Fords zur Förderung der wissenschaftlichen Forschung), Austrian Science Fund, Project No. P21636-B18.

\section{References}

[1] C. Baumgartner, Handbuch der Epilepsien: Klinik, Diagnostik, Therapien und Psychosoziale Aspekte, Springer, Wien, Austria, 2001.

[2] N. Bernasconi, A. Bernasconi, Z. Caramanos, S. B. Antel, F. Andermann, and D. L. Arnold, "Mesial temporal damage in temporal lobe epilepsy: a volumetric MRI study of the hippocampus, amygdala and parahippocampal region," Brain, vol. 126, no. 2, pp. 462-469, 2003.

[3] S. S. Keller and N. Roberts, "Voxel-based morphometry of temporal lobe epilepsy: an introduction and review of the literature," Epilepsia, vol. 49, no. 5, pp. 741-757, 2008.

[4] N. Bernasconi, S. Duchesne, A. Janke, J. Lerch, D. L. Collins, and A. Bernasconi, "Whole-brain voxel-based statistical analysis of gray matter and white matter in temporal lobe epilepsy," NeuroImage, vol. 23, no. 2, pp. 717-723, 2004.

[5] L. Rocha, L. Lorigados-Pedre, S. Orozco-Suárez et al., "Autoradiography reveals selective changes in serotonin binding in neocortex of patients with temporal lobe epilepsy," Progress in Neuro-Psychopharmacology and Biological Psychiatry, vol. 31, no. 6, pp. 1208-1218, 2007.

[6] S. Takaya, T. Hanakawa, K. Hashikawa et al., "Prefrontal hypofunction in patients with intractable mesial temporal lobe epilepsy," Neurology, vol. 67, no. 9, pp. 1674-1676, 2006.

[7] C. P. Chang, D. J. Yen, S. M. Yu et al., "Unilateral thalamic hypometabolism in patients with temporal lobe epilepsy," Journal of the Formosan Medical Association, vol. 107, no. 7, pp. 567-571, 2008.

[8] F. Semah, "Temporoporal metabolic abnormalities in temporal lobe epilepsies," Epileptic Disorders, vol. 4, no. 1, pp. S41S49, 2002.

[9] G. E. Alexander, M. R. DeLong, and P. L. Strick, "Parallel organization of functionally segregated circuits linking basal ganglia and cortex," Annual Review of Neuroscience, vol. 9, pp. 357-381, 1986.

[10] M. S. Mega and J. L. Cummings, "Frontal-subcortical circuits and neuropsychiatric disorders," Journal of Neuropsychiatry and Clinical Neurosciences, vol. 6, no. 4, pp. 358-370, 1994.

[11] O. Godefroy, "Frontal syndrome and disorders of executive functions," Journal of Neurology, vol. 250, no. 1, pp. 1-6, 2003.

[12] B. Hanna-Pladdy, "Dysexecutive syndromes in neurologic disease," Journal of Neurologic Physical Therapy, vol. 31, no. 3, pp. 119-127, 2007. 
[13] A. M. Owen, J. J. Downes, B. J. Sahakian, C. E. Polkey, and T. W. Robbins, "Planning and spatial working memory following frontal lobe lesions in man," Neuropsychologia, vol. 28, no. 10, pp. 1021-1034, 1990.

[14] T. Shallice, "Specific impairments of planning," Philosophical Transactions of the Royal Society of London. Series B, vol. 298, no. 1089, pp. 199-209, 1982.

[15] F. Collette, M. van der Linden, S. Laureys et al., "Exploring the unity and diversity of the neural substrates of executive functioning," Human Brain Mapping, vol. 25, no. 4, pp. 409423, 2005.

[16] J. D. Schmahmann and J. C. Sherman, "The cerebellar cognitive affective syndrome," Brain, vol. 121, no. 4, pp. 561$579,1998$.

[17] T. Benke, M. Delazer, L. Bartha, and A. Auer, "Basal ganglia lesions and the theory of fronto-subcortical loops: neuropsychological findings in two patients with left caudate lesions," Neurocase, vol. 9, no. 1, pp. 70-85, 2003.

[18] Y. D. van der Werf, P. Scheltens, J. Lindeboom, M. P. Witter, H. B. M. Uylings, and J. Jolles, "Deficits of memory, executive functioning and attention following infarction in the thalamus; a study of 22 cases with localised lesions," Neuropsychologia, vol. 41, no. 10, pp. 1330-1344, 2003.

[19] M. Schacher, R. Winkler, T. Grunwald et al., "Mesial temporal lobe epilepsy impairs advanced social cognition," Epilepsia, vol. 47, no. 12, pp. 2141-2146, 2006.

[20] C. Helmstaedter and E. Kockelmann, "Cognitive outcomes in patients with chronic temporal lobe epilepsy," Epilepsia, vol. 47, no. 2, pp. 96-98, 2006.

[21] S. Meletti, F. Benuzzi, G. Cantalupo, G. Rubboli, C. A. Tassinari, and P. Nichelli, "Facial emotion recognition impairment in chronic temporal lobe epilepsy," Epilepsia, vol. 50, no. 6, pp. 1547-1559, 2009.

[22] I. D. S. S. Tudesco, L. J. Vaz, M. A. S. Mantoan et al., "Assessment of working memory in patients with mesial temporal lobe epilepsy associated with unilateral hippocampal sclerosis," Epilepsy and Behavior, vol. 18, no. 3, pp. 223-228, 2010.

[23] C. Helmstaedter, "Effects of chronic epilepsy on declarative memory systems," Progress in Brain Research, vol. 135, pp. 439-453, 2002.

[24] K. Labudda, K. Frigge, S. Horstmann et al., "Decision making in patients with temporal lobe epilepsy," Neuropsychologia, vol. 47, no. 1, pp. 50-58, 2009.

[25] M. Delazer, L. Zamarian, E. Bonatti et al., "Decision making under ambiguity and under risk in mesial temporal lobe epilepsy," Neuropsychologia, vol. 48, no. 1, pp. 194-200, 2010.

[26] A. R. Giovagnoli, "Relation of sorting impairment to hippocampal damage in temporal lobe epilepsy," Neuropsychologia, vol. 39, no. 2, pp. 140-150, 2001.

[27] C. H. Kim, S. A. Lee, H. J. Yoo, J. K. Kang, and J. K. Lee, "Executive performance on the Wisconsin card sorting test in mesial temporal lobe epilepsy," European Neurology, vol. 57, no. 1, pp. 39-46, 2007.

[28] R. Corcoran and D. Upton, "A role for the hippocampus in card sorting?” Cortex, vol. 29, no. 2, pp. 293-304, 1993.

[29] S. Lehrl, Manual zum MWT-B, Spitta, Balingen, Germany, 1991.

[30] I. M. Lipkus, G. Samsa, and B. K. Rimer, "General performance on a numeracy scale among highly educated samples," Medical Decision Making, vol. 21, no. 1, pp. 37-44, 2001.

[31] E. Peters, D. Västfjäll, P. Slovic, C. K. Mertz, K. Mazzocco, and S. Dickert, "Numeracy and decision making," Psychological Science, vol. 17, no. 5, pp. 407-413, 2006.
[32] M. Jackson and E. K. Warrington, "Arithmetic skills in patients with unilateral cerebral lesions," Cortex, vol. 22, no. 4, pp. 611620, 1986.

[33] J. Ilmberger, “Der Münchner Gedachtnistest,” 1988.

[34] D. Schellig and B. Chächtele, Visueller unf Verbaler Merkfahigkeitstest (VVM), Pearson Assessment \& Information GmbH, Frankfurt am Main, Germany, 2009.

[35] C. Hermann, U. Buss, and R. P. Snaith, Hospital Anxiety and Depression Scale - German (HADS-D), Hans Huber, Bern, Switzerland, 1995.

[36] C. Härting, H. J. Markowitsch, H. Neufeld, P. Calabrese, H. Deisinger, and J. Kessler, WMS-R Wechsler GedachtnistestRevidierte Fassung, Hans Huber, Bern, Switzerland, 2000.

[37] M. D. Lezak, Neuropsychological Assessment, Oxford University Press, New York, NY, USA, 1998.

[38] S. Aschenbrenner, O. Tucha, and K. W. Lange, Regensburger Wortflüssigkeits-Test (RWT), Hogrefe, Göttingen, Germany, 2000.

[39] S. K. Kongs, L. L. Thompson, G. L. Iverson, and R. K. Heaton, The Wisconsin Card Sorting Test (WCST-64), Hogrefe Testzentrale, Göttingen, Germany, 2000.

[40] A. M. Fox, P. T. Michie, C. D. L. Wynne, and M. T. Maybery, "ERP correlates of response inhibition to elemental and configural stimuli in a negative patterning task," Clinical Neurophysiology, vol. 111, no. 6, pp. 1045-1053, 2000.

[41] J. Kohler and U. Beck, Planungstest, Beck \& Kohler GbR, Kostanz, Germany, 2000.

[42] R. K. Heaton, G. L. Chelune, J. L. Talley, G. G. Kay, and G. Cutiss, Wisconsin Card Sorting Test Manual, Psychological Assessment Resources, Odessa, Fla, USA, 1993.

[43] R. C. Martin, S. M. Sawrie, F. G. Gilliam et al., "Wisconsin card sorting performance in patients with temporal lobe epilepsy: clinical and neuroanatomical correlates," Epilepsia, vol. 41, no. 12 , pp. 1626-1632, 2000.

[44] D. Upton and R. Corcoran, "The role of the right temporal lobe in card sorting: a case study," Cortex, vol. 31, no. 2, pp. 405-409, 1995.

[45] L. C. Black, B. K. Schefft, S. R. Howe, J. P. Szaflarski, H. S. Yeh, and M. D. Privitera, "The effect of seizures on working memory and executive functioning performance," Epilepsy and Behavior, vol. 17, no. 3, pp. 412-419, 2010.

[46] K. J. Meador, "Cognitive and memory effects of the new antiepileptic drugs," Epilepsy Research, vol. 68, no. 1, pp. 6367, 2006

[47] A. P. Aldenkamp, M. De Krom, and R. Reijs, "Newer antiepileptic drugs and cognitive issues," Epilepsia, vol. 44, no. 4, pp. 21-29, 2003.

[48] A. E. Cavanna, F. Ali, H. E. Rickards, and D. McCorry, "Behavioral and cognitive effects of anti-epileptic drugs," Discovery medicine, vol. 9, no. 45, pp. 138-144, 2010.

[49] E. Hessen, M. I. Lossius, and L. Gjerstad, "Antiepileptic monotherapy significantly impairs normative scores on common tests of executive functions," Acta Neurologica Scandinavica, vol. 119, no. 3, pp. 194-198, 2009.

[50] R. C. Oldfield, "The assessment and analysis of handedness: the Edinburgh inventory," Neuropsychologia, vol. 9, no. 1, pp. 97-113, 1971.

[51] K. Nearing, D. Madhavan, and O. Devinsky, "Temporal lobe epilepsy: a progressive disorder?" Reviews in Neurological Diseases, vol. 4, no. 3, pp. 122-127, 2007.

[52] S. Lee, V. Sziklas, F. Andermann et al., "The effects of adjunctive topiramate on cognitive function in patients with epilepsy," Epilepsia, vol. 44, no. 3, pp. 339-347, 2003. 
[53] B. Hermann and M. Seidenbergs, "Executive system dysfunction in temporal lobe epilepsy: effects of nociferous cortex versus hippocampal pathology," Journal of Clinical and Experimental Neuropsychology, vol. 17, no. 6, pp. 809-819, 1995.

[54] H. Barbas, "Connections underlying the synthesis of cognition, memory, and emotion in primate prefrontal cortices," Brain Research Bulletin, vol. 52, no. 5, pp. 319-330, 2000.

[55] S. Colnat-Coulbois, K. Mok, D. Klein, S. Pénicaud, T. Tanriverdi, and A. Olivier, "Tractography of the amygdala and hippocampus: anatomical study and application to selective amygdalohippocampectomy," Journal of Neurosurgery, vol. 113, no. 6, pp. 1135-1143, 2010.

[56] A. Miyake, N. P. Friedman, M. J. Emerson, A. H. Witzki, A. Howerter, and T. D. Wager, "The unity and diversity of executive functions and their contributions to complex "Frontal Lobe" tasks: a latent variable analysis," Cognitive Psychology, vol. 41, no. 1, pp. 49-100, 2000.

[57] A. D. Baddeley, "Is working memory still working?" American Psychologist, vol. 56, no. 11, pp. 851-864, 2001.

[58] F. Collette and M. van der Linden, "Brain imaging of the central executive component of working memory," Neuroscience and Biobehavioral Reviews, vol. 26, no. 2, pp. 105-125, 2002.

[59] A. Baddeley, "Working memory," Science, vol. 255, no. 5044, pp. 556-559, 1992. 


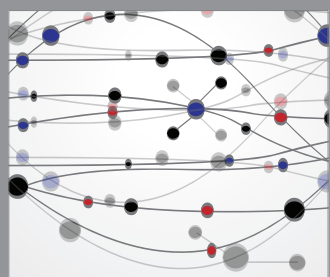

The Scientific World Journal
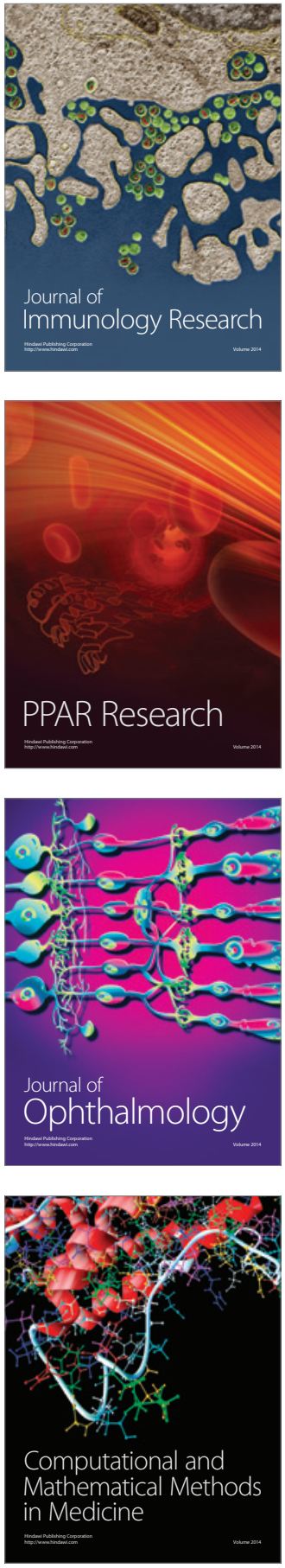

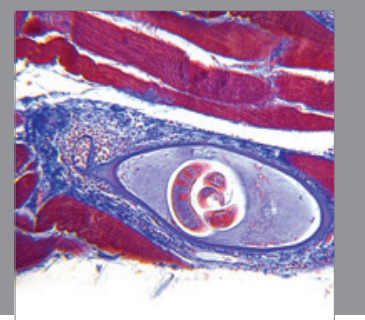

Gastroenterology

Research and Practice
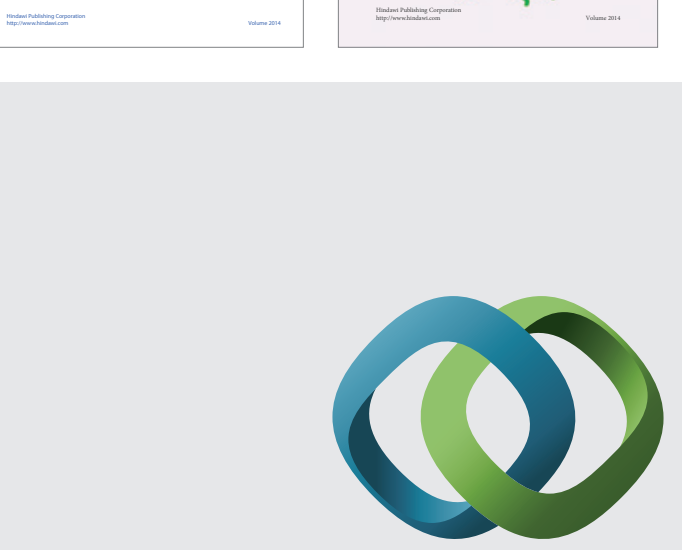

\section{Hindawi}

Submit your manuscripts at

http://www.hindawi.com
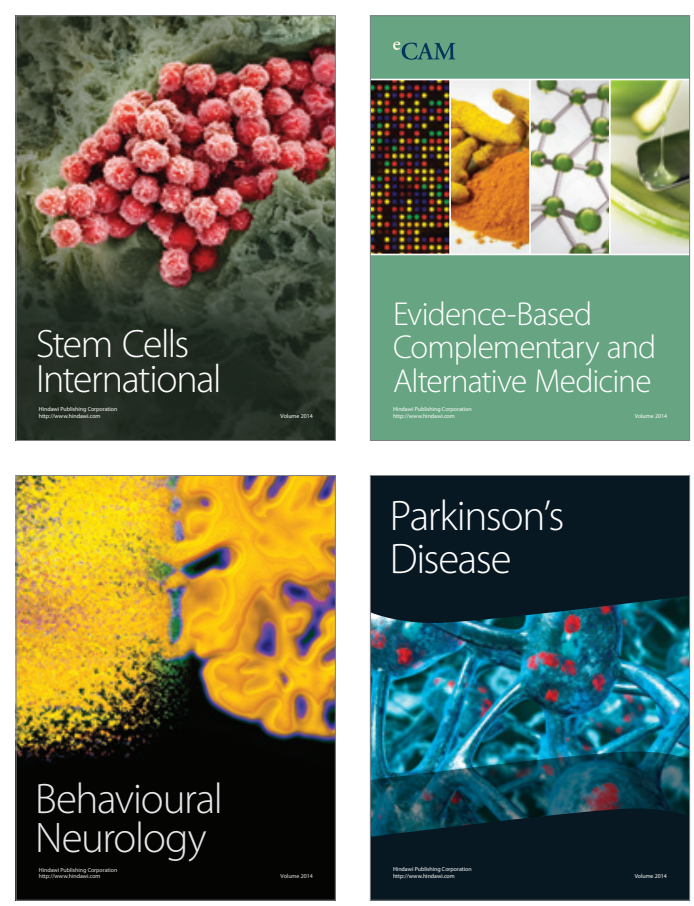

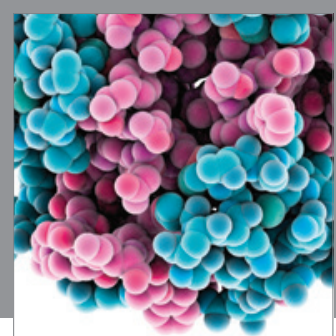

Journal of
Diabetes Research

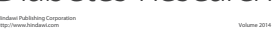

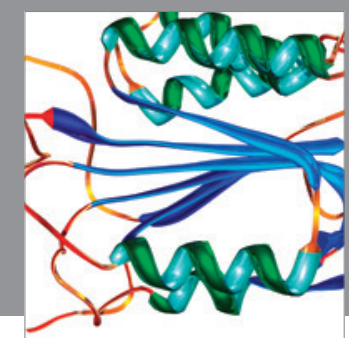

Disease Markers
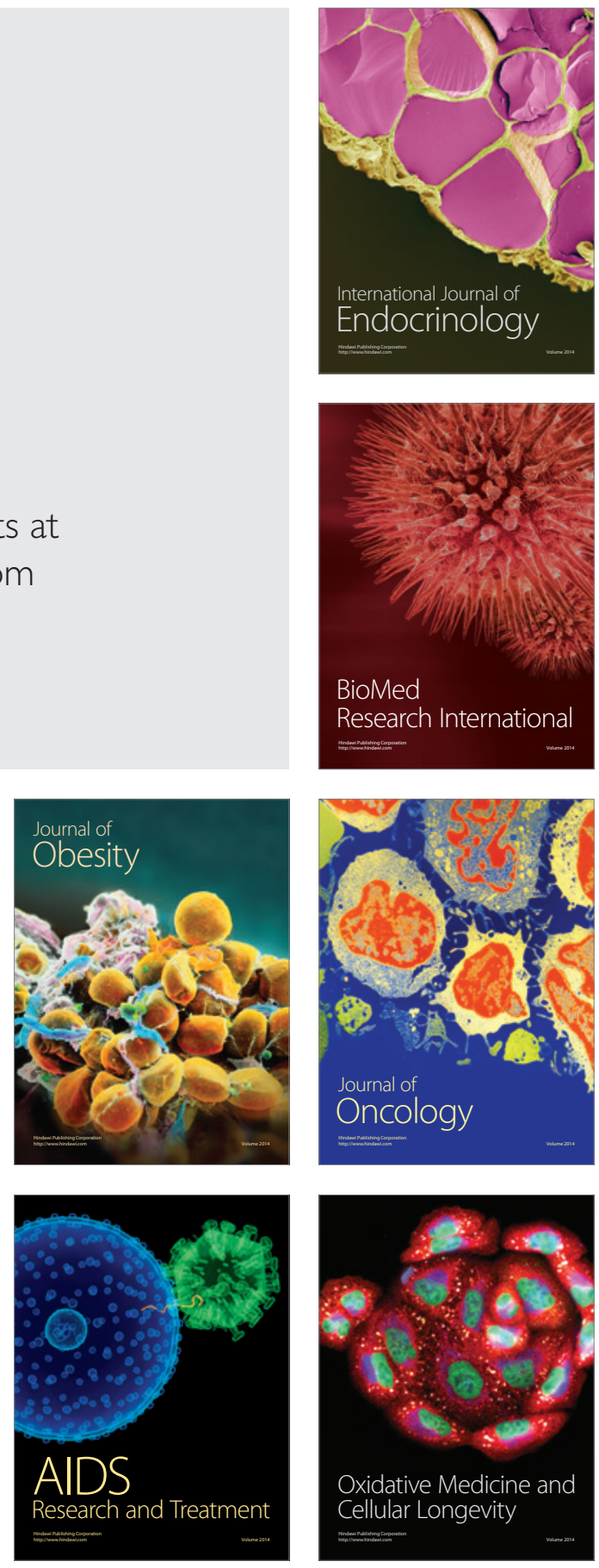